\title{
LINEAR SOLUTION FOR GENERATION OF TSUNAMI WAVES WITH GROUND MOTION AND TIMESCALES
}

\begin{abstract}
Marine Le Gal ${ }^{1}$, Damien Violeau ${ }^{2}$, Michel Benoit ${ }^{3}$
Tsunami generation have commonly been modeled by using the Okada method (see Okada (1992)), i.e. from seismic data, the final ground deformation is calculated and applied to the free surface as an initial deformation. Using this method, lot of aspects of the generation are neglected, including timescales. The aim of this study is to measure the influence of temporal parameters during a simplified kinematic generation. In this purpose, a junction between the work of Hammack (1973) and Todorovska and Trifunac (2001) is done to built a linear semi-analytical solution of the free surface deformation depending simultaneously of the the rise time, $t_{r}$, and the rupture velocity, $v_{p}$. They characterize the vertical and horizontal motions respectively. This solution is compared and validated with numerical simulations and the influence of the temporal parameters is measured by varying their values in a large range. A resonance phenomenon appears, as noticed by Todorovska and Trifunac (2001), but only for short rise times. It manifests an amplification of the generated wave amplitude against the ground deformation amplitude at the end of the motion. Then, using numerical simulations, this conclusion is briefly extended to the nonlinear regime.
\end{abstract}

Keywords: tsunami wave generation; timescales; linear theory

\section{INTRODUCTION}

Most of tsunamis are generated by earthquakes, i.e. kinematic seafloor deformations. However, the traditionnal way to model the generation of a seismic tsunami is to estimate the ground deformation with the calculation method from Okada (1992) and to transfer instantaneously the final vertical deformation to the free surface. With this method, some aspects of the generation are not included as recalled by Nosov (2014), among them the kinematic aspect of the ground deformation. Even if this approximation is generally acceptable, some events, called "Tsunami earthquakes" by Kanamori (1972), involve slow dynamics which can impact the form and properties of the generated wave. Kinematic generation has already been studied in the past for real case events, as done by Dutykh et al. (2013), or idealised ground motions. Generally, the idealised displacement depends on a single temporal parameter while two can be defined: the rise time $t_{r}$ and the rupture velocity $v_{p}$, that represent the vertical and horizontal timescales respectively. The major contributions about these parameters were done by Hammack (1973) for the rise time and Todorovska and Trifunac (2001) for the rupture velocity. The present study is built on their work. First, a semi-analytical solution of the free surface deformation for a linear idealised ground displacement is developped with the aim of measuring the influence of $t_{r}$ and $v_{p}$. A cross validation is performed with numerical results obtained from the linear version of the code Misthyc and an amplification ratio of the free surface against the sea floor deformations is defined. This linear analysis emphasizes a resonance phenomenon at certain $t_{r}$ and $v_{p}$ for which the amplification ratio reaches large values. Then, this behaviour is briefly numerically generalised to the nonlinear regime.

\section{LINEAR THEORETICAL MODEL}

The considered fluid domain is represented in Figure 1a. The analysis is restricted here to one unbounded horizontal direction $x$. The Coriolis and surface tension effects are neglected. The fluid is assumed incompressible and inviscid. It is delimited by the free surface $z=\eta(x, t)$ and the sea floor $z=-h+\zeta(x, t)$, where $h$ is the uniform depth and $\zeta$ the sea floor deformation.

Initially, the fluid is at rest with $\eta(x, t=0)=0$ and $\zeta(x, t=0)=0$. To simplify the problem, the ground motion is reduced to an uplift of a rectangular block which propagates with a given rupture velocity $v_{p}$ along the $x$ axis for $x \in[0, L], L$ being the deformation length. The vertical deformation reaches the maximal amplitude $\zeta_{0}$ in a finite time, the rise time $t_{r}$, along the $z$ axis. Figure $1 \mathrm{~b}$ gives a schematic representation of this motion. Building on the previous work of Hammack (1973) and Todorovska and Trifunac (2001), that only considered one temporal parameter, the deformation is defined here as:

$$
\begin{aligned}
\zeta(x, t) & =\zeta_{0} H(L-x) H(x) \mathcal{T}(x, t) \\
\mathcal{T}(x, t) & =H\left(t-\frac{x}{v_{p}}\right) H\left(\frac{x}{v_{p}}+t_{r}-t\right) \frac{1}{2}\left(1-\cos \omega_{r}\left(t-\frac{x}{v_{p}}\right)\right)+H\left(t-\frac{x}{v_{p}}-t_{r}\right),
\end{aligned}
$$

\footnotetext{
${ }^{1}$ Saint-Venant Laboratory for Hydraulics, Joint research unit EDF - Cerema - Ecole des Ponts, Chatou, France

${ }^{2}$ EDF \& Saint-Venant Laboratory for Hydraulics, Joint research unit EDF - Cerema - Ecole des Ponts, Chatou, France

${ }^{3}$ Institut de Recherche sur les Phénomènes Hors Equilibre (IRPHE), UMR 7342, 13384 Marseille Cedex 13, France
} 


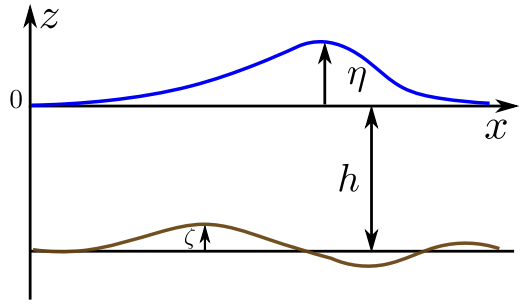

(a)

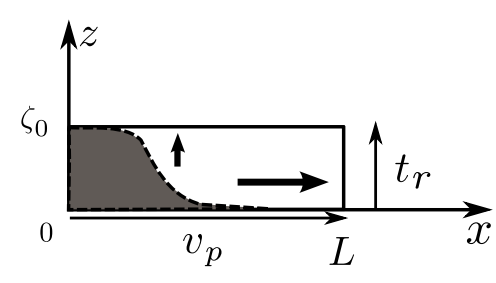

(b)

Figure 1: 1a: Definition of the considered domain and coordinate system. $h$ is the uniform water depth, $\zeta(x, t)$ and $\eta(x, t)$ are the sea floor and free surface deformations, respectively. 1b: Definition of the kinematic sea floor deformation with $\zeta_{0}$ the amplitude of the deformation, $v_{p}$ the rupture velocity, $t_{r}$ the rise time and $L$ the deformation length. The grey zone is transitional while the solid lines represent the final state.

with $\omega_{r}=\pi / t_{r}$ and $H$ is the heaviside function. If $t_{r}=0 \mathrm{~s}$, the expression corresponds to the $1 \mathrm{D}$ definition given by Todorovska and Trifunac (2001), and if the limit is taken for $v_{p} \rightarrow \infty$, the definition of Hammack (1973) is recovered.

The flow is irrotational, thus there exists a velocity potential $\phi(x, t)$ which satisfies the Laplace equation and the boundary conditions. Under the assumption of small perturbations, the problem is linearized as follows:

$$
\begin{array}{cc}
\nabla^{2} \phi=0, & \\
\phi_{z}=\eta_{t} & z=0, \\
\phi_{t}+g \eta=0 & z=0, \\
\phi_{z}=\zeta_{t} & z=-h,
\end{array}
$$

where subscripts $t$ and $x$ indicate partial derivatives.

The Fourier-Laplace transform is introduced as:

$$
\begin{gathered}
\mathcal{F} \mathcal{L}(f)=\bar{f}(k, s)=\int_{\mathfrak{R}} e^{-i k x} d x \int_{0}^{+\infty} f(x, t) e^{-s t} d t \\
\mathcal{F} \mathcal{L}^{-1}(\bar{f})=f(x, t)=\frac{1}{2 \pi} \int_{\mathfrak{R}} e^{i k x} \frac{1}{i 2 \pi} \int_{\gamma-i \infty}^{\gamma+i \infty} \bar{f}(k, s) e^{s t} d k d s .
\end{gathered}
$$

where $\gamma$ is a real constant that insures the existence of the integral. This transform is applied to the system 3 6 giving:

$$
\begin{aligned}
\bar{\phi}_{z z}-k^{2} \bar{\phi}=0, & \\
\bar{\phi}_{z}=-\frac{s^{2}}{g} \bar{\phi} & z=0, \\
\bar{\phi}_{z}=s \bar{\zeta} & z=-h, \\
s \bar{\phi}=-g \bar{\eta} & z=0 .
\end{aligned}
$$

An analytical solution of Eq. $9-11$ in $(k, s)$ space is found as:

$$
\bar{\phi}(k, z, s)=\frac{-s g \bar{\zeta}(k, s)}{\left(s^{2}+\omega^{2}\right) \cosh k h}\left[\cosh k z-\frac{s^{2}}{g k} \sinh k z\right],
$$

thus from Eq. 12,

$$
\bar{\eta}(k, s)=\frac{s^{2} \bar{\zeta}(k, s)}{\left(s^{2}+\omega^{2}\right) \cosh k h},
$$

where $\omega^{2}=g k \tanh k h$ is the linear dispersion relation. Using the transform Eq. 7 with the definition of the sea floor deformation Eq. 2, we obtain the analytical solution in this particular case:

$$
\bar{\zeta}(k, s)=\frac{\zeta_{0}}{2}\left(1+e^{-s t_{r}}\right) \frac{\omega_{r}^{2}}{s\left(s^{2}+\omega_{r}^{2}\right)} \frac{1-e^{-L\left(i k+\frac{s}{v_{p}}\right)}}{i k+\frac{s}{v_{p}}},
$$




$$
\bar{\eta}(k, s)=\frac{\zeta_{0}}{2} \frac{s}{\left(s^{2}+\omega^{2}\right) \cosh k h}\left(1+e^{-s t_{r}}\right) \frac{\omega_{r}^{2}}{s^{2}+\omega_{r}^{2}} \frac{1-e^{-L\left(i k+\frac{s}{v_{p}}\right)}}{i k+\frac{s}{v_{p}}} .
$$

To come back to the physical space $(x, t)$, first an analytical inverse Lapace transform is performed on Eq. 16 giving an analytical Fourier transform of the free surface deformation:

$$
\mathcal{F}(\eta)(k, t)=\frac{\zeta_{0}}{2} \frac{v_{p}}{\cosh k h} \frac{\omega_{r}^{2}}{\omega_{r}^{2}-\omega^{2}}\left(\begin{array}{l}
f(k, t) \\
+H\left(t-t_{r}\right) f\left(k, t-t_{r}\right) \\
-H\left(t-\frac{L}{v_{p}}\right) e^{-i k L} f\left(k, t-\frac{L}{v_{p}}\right) \\
-H\left(t-t_{r}-\frac{L}{v_{p}}\right) e^{-i k L} f\left(k, t-t_{r}-\frac{L}{v_{p}}\right)
\end{array}\right),
$$

with:

$$
\begin{aligned}
f(k, t)= & \frac{1}{\omega^{2}-k^{2} v_{p}^{2}}\left(i k v_{p} \cos (\omega t)+\omega \sin (\omega t)-i k v_{p} e^{-i k v_{p} t}\right) \\
- & \frac{1}{\omega_{r}^{2}-k^{2} v_{p}^{2}}\left(i k v_{p} \cos \left(\omega_{r} t\right)+\omega_{r} \sin \left(\omega_{r} t\right)-i k v_{p} e^{-i k v_{p} t}\right) .
\end{aligned}
$$

Then, a numerical integration with Simpson's method is used to calculate the free surface deformation (the inverse Fourier transform of Eq. 17, as a closed form is too complex to obtain.

$$
\eta(x, t)=\frac{1}{2 \pi} \int_{R} e^{i k x} \mathcal{F}(\eta) d k .
$$

From now, only dimensionless notations, annoted with *, will be used. The dimensionless variables are defined as follows:

$$
\eta^{*}=\frac{\eta}{h}, \quad t^{*}=t \sqrt{\frac{g}{h}}, \quad x^{*}=\frac{x}{h}, \quad \zeta^{*}=\frac{\zeta}{h}
$$

and the parameters become:

$$
\tau^{*}=\frac{t_{r}}{L} \sqrt{g h}, \quad v_{p}^{*}=\frac{v_{p}}{\sqrt{g h}}, \quad L^{*}=\frac{L}{h} .
$$

The dimensionless temporal parameters, $\tau^{*}$ and $v_{p}^{*}$, have already been defined by Hammack (1973) and Todorovska and Trifunac (2001), respectively.

\section{INFLUENCE OF THE TIMESCALES}

The linear solution derived in the previous section is now used to study the influence of $\tau^{*}$ and $v_{p}^{*}$ on the wave trains generated by the kinematic sea floor deformation Eq. 2. For this purpose, the semi-analytical solution Eq. 19 is calculated for a large panel of their values.

\section{Theoretical free surface deformation at the end of the sea floor motion}

By convention, we define the end of the generation period, and thus the beginning of the propagation one, when the ground stops moving, i.e. at $t^{*}=t_{r}^{*}+L^{*} / v_{p}^{*}$. For this study, the deformation length is kept constant as $L^{*}=50$. Also, the value of the deformation's amplitude $\zeta_{0}^{*}$ is considered minor since the model is linear (linear solution proportional to it), thus the result will show the ratio $\eta^{*} / \zeta_{0}^{*}$. The rupture velocity $v_{p}^{*}$ varies between 0.5 and 50 , and the rise time $\tau^{*}$ between 0 and 5 .

The spatial profiles of the solution for a sample of these values are compared in Figure 2 with those given by the numerical program Misthyc, which solves the Zakharov equations (see Zakharov (1968)). The choice of the equations is leaded by its capacity to well represent the nonlinearity and the dispersion of wave propagation. Misthyc proved very efficient in predicting nonlinear or linear dispersive water wave (Yates and Benoit (2015)). The code is used here in a linear mode, corresponding to solving the system 3-6. This comparison validates the theoretical solution as the curves are almost superimposed.

The maximum free surface elevation $\eta_{\max }^{*} / \zeta_{0}^{*}$ varies with $v_{p}^{*}$ and $\tau^{*}$. If $v_{p}^{*}=50$ (i.e. very large) and $\tau^{*}=0$, the free surface deformation is almost identical to the sea floor deformation (i.e. $\eta_{\text {max }}^{*} / \zeta_{0}^{*} \simeq 1$ ) since the deformation is nearly instantaneous in both horizontal and vertical directions. When $\tau^{*}$ increases, the wave begins to propagate before the end of the ground motion as noted by Jamin et al. (2015), the wave amplitude is smaller, and the wave propagates in both directions $( \pm x)$. When $v_{p}^{*}$ decreases, an asymmetry appears: the 


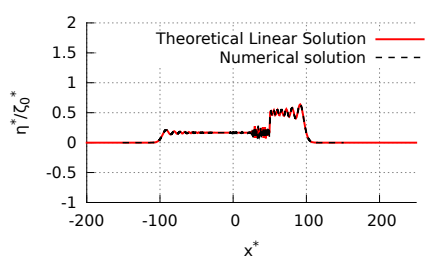

(a) $v_{p}^{*}=0.5 ; \tau^{*}=0$

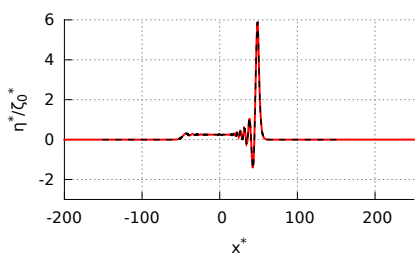

(d) $v_{p}^{*}=1 ; \tau^{*}=0$

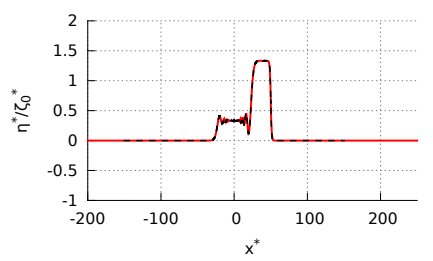

(g) $v_{p}^{*}=2 ; \tau^{*}=0$

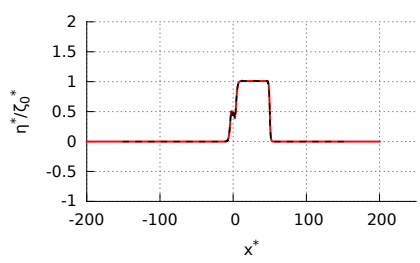

(j) $v_{p}^{*}=10 ; \tau^{*}=0$

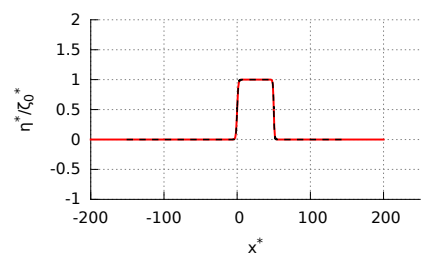

(m) $v_{p}^{*}=50 ; \tau^{*}=0$

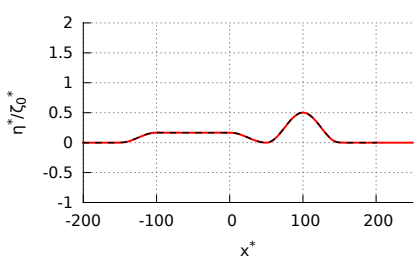

(b) $v_{p}^{*}=0.5 ; \tau^{*}=1$

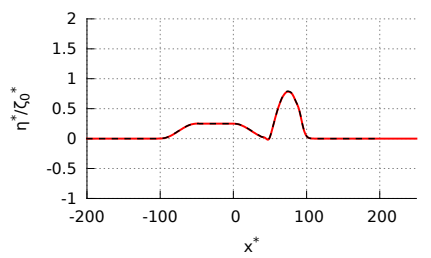

(e) $v_{p}^{*}=1 ; \tau^{*}=1$

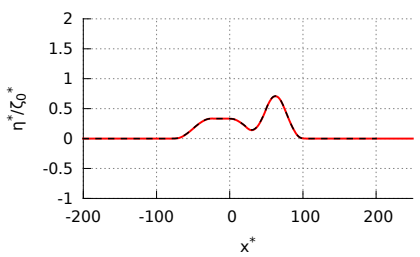

(h) $v_{p}^{*}=2 ; \tau^{*}=1$

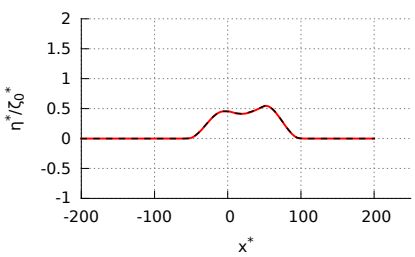

(k) $v_{p}^{*}=10 ; \tau^{*}=1$

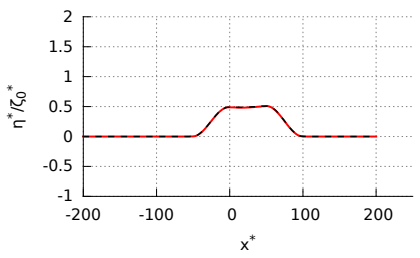

(n) $v_{p}^{*}=50 ; \tau^{*}=1$

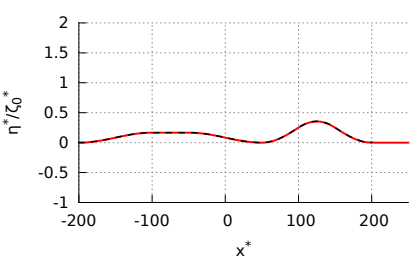

(c) $v_{p}^{*}=0.5 ; \tau^{*}=2$

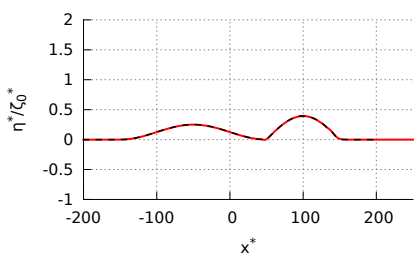

(f) $v_{p}^{*}=1 ; \tau^{*}=2$

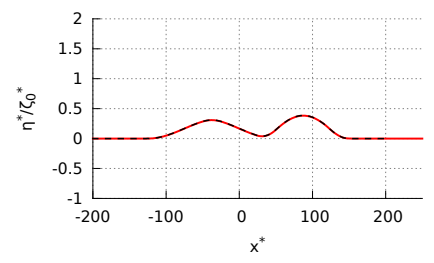

(i) $v_{p}^{*}=2 ; \tau^{*}=2$

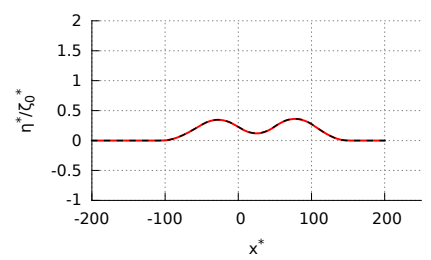

(l) $v_{p}^{*}=10 ; \tau^{*}=2$

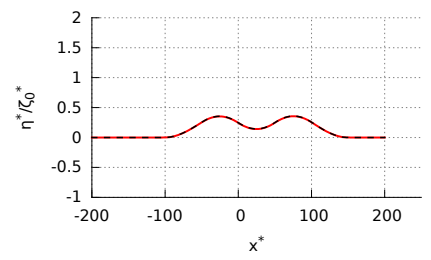

(o) $v_{p}^{*}=50 ; \tau^{*}=2$

Figure 2: Free surface profiles at $t^{*}=t_{r}^{*}+L^{*} / v_{p}^{*}$ for $L^{*}=50$ and different values of $v_{p}^{*}$ and $\tau^{*}\left(v_{p}^{*}\right.$ increases from top to bottom and $\tau^{*}$ increases from left to right). The red line is the linear solution Eq. 19, and the dashed black line is the numerical simulation results from the linear version of the Misthyc code. The vertical scale of graph (d) for $v_{p}^{*}=1$ and $\tau^{*}=0$ differs from the others for clarity.

wave propagating in the same direction as the ground deformation $(+x)$ is larger.

Figure 3 shows the dependency of $\eta_{\max }^{*} / \zeta_{0}^{*}$ on the temporal parameters in $v_{p}^{*}-\tau^{*}$ space. For small $\tau^{*}$, the parameter $v_{p}^{*}$ has a strong influence on the maximum amplitude. As shown by Todorovska and Trifunac (2001), a resonance appears for $v_{p}^{*}$ in the range 0.7 to 2 (i.e. close to $v_{p}^{*}=1$ ) generating a wave that reaches an amplitude $\eta_{\max }^{*} / \zeta_{0}^{*}$ larger than 1 . The maximum free surface amplitude can reach six times the bottom deformation amplitude at $v_{p}^{*}=1$. Thus, when the rupture velocity is close to the wave velocity, the amplitude of the generated wave is much larger than the amplitude of the sea floor deformation at the beginning 


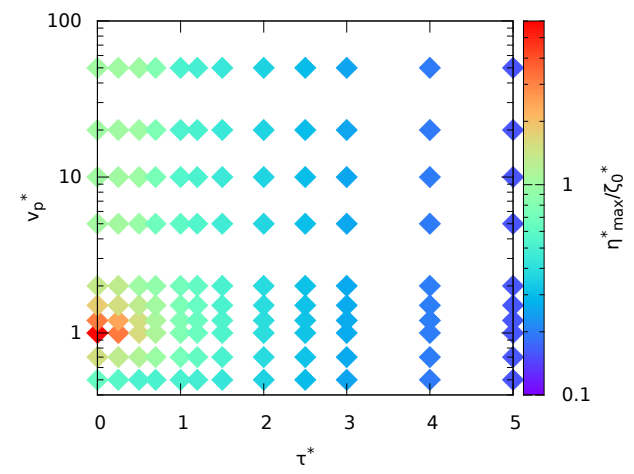

Figure 3: The maximum free surface amplitude, $\eta_{\max }^{*} / \zeta_{0}^{*}$ as a function of $v_{p}^{*}$ and $\tau^{*}$ for $L^{*}=50$ at $t^{*}=$ $t_{r}^{*}+L^{*} / v_{p}^{*}$ (colour in log scale).

of the propagation phase. However, this resonance only exists for small values of $\tau^{*}$.

The deformation's length is then varied in the particular case of $v_{p}^{*}=1$ and $\tau^{*}=0$. In Figure 4 , the ratio $\eta_{\max }^{*} / \zeta_{0}^{*}$ is plotted as a function of $L^{*}$.

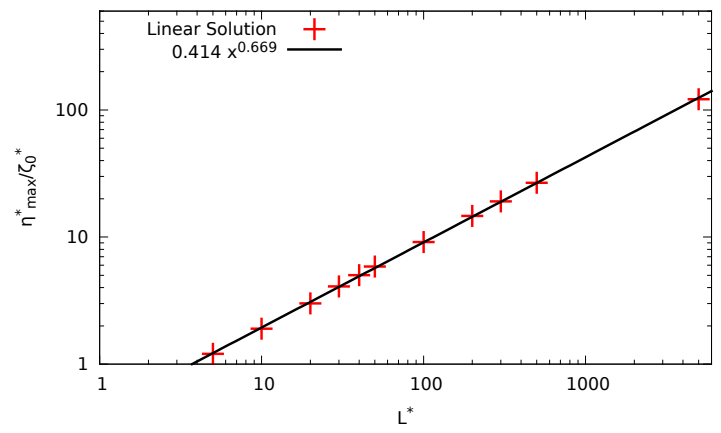

Figure 4: Impact of the deformation's length with $v_{p}^{*}=1$ and $\tau^{*}=0$. The red crosses are the ratios $\eta_{\text {max }}^{*} / \zeta_{0}^{*}$ and the black line is the trend line $0.414\left(L^{*}\right)^{0.669}$.

The maximum amplitude of the deformation is larger for larger $L^{*}$. This is consistent with the fact that a larger volume of water is displaced. The maxima can be reasonably well fitted with a power law of the form:

$$
\eta_{\max }^{*} / \zeta_{0}^{*}=0.414\left(L^{*}\right)^{0.669} \quad \text { for } \quad L^{*} \in[0 ; 5000] \text {. }
$$

\section{Propagation stage}

The propagation of the generated wave is analysed as a function of $v_{p}^{*}$ and $\tau^{*}$, with fixed $L^{*}=50$. Similarly to what was done at the end of the ground motion, the theoretical free surface deformation is estimated for larger times and the spectrum of the ratio $\eta_{\max }^{*} / \zeta_{0}^{*}$ is calculated at $t^{*}=100,500$ and 1000 as shown in Figure 5. The shape of the dependence does not change with time, except for small $v_{p}^{*}$ and large $\tau^{*}$ because the end of the ground motion occurs later than $t^{*}=100$. However, the ratio decreases in time around $v_{p}^{*}=1$ and small $\tau^{*}$, where the amplitudes are greater than 1 , thus resonance is preserved for these times but the amplification may disappear after a while. Figure 6 shows the evolution of the free surface deformation $\eta^{*} / \zeta_{0}^{*}$ as a function of $x$ and $t$ for $v_{p}^{*}=1$ and $\tau^{*}=0$. The wave asymmetry is clearly visible. 


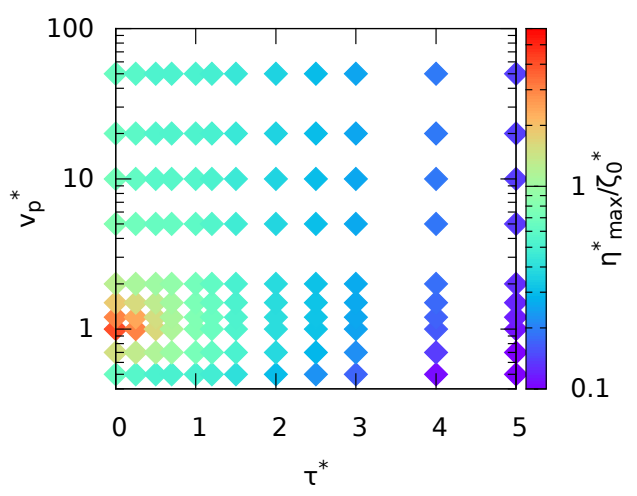

(a) $t^{*}=100$

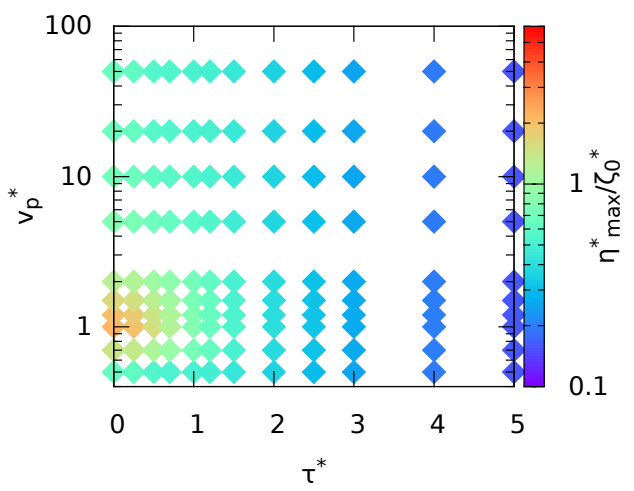

(b) $t^{*}=500$

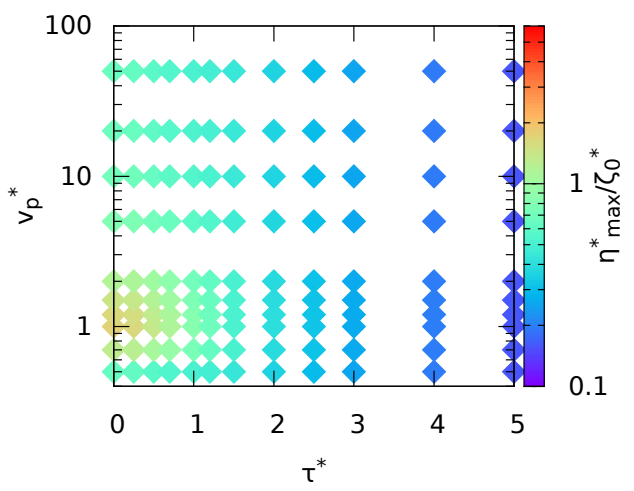

(c) $t^{*}=1000$

Figure 5: Maximum free surface amplitude $\eta_{\max }^{*} / \zeta_{0}^{*}$ as a function of $v_{p}^{*}$ and $\tau^{*}$ for $L^{*}=50$ at different times $t^{*}$. (colour in log scale).

Moreover, a dispersion phenomenon appears in the $+x$ direction, explaining the decrease of the maximal wave amplitude. 


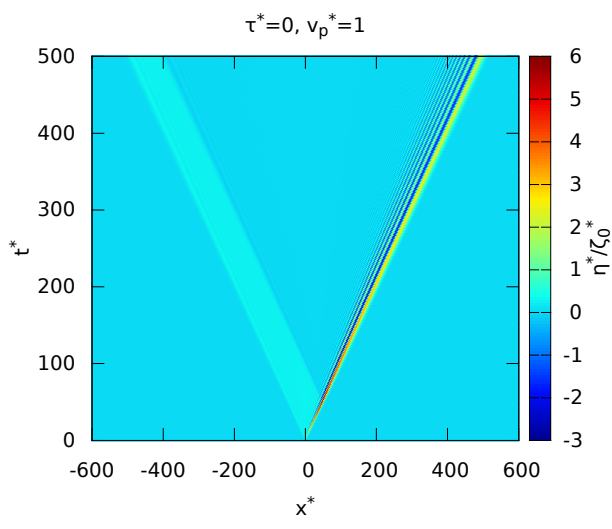

Figure 6: Evolution in space and time of the free surface deformation $\eta^{*} / \zeta_{0}^{*}$ for $v_{p}^{*}=1$ and $\tau^{*}=0$. The colour represents the normalized wave amplitude.

\section{NUMERICAL SIMULATION OF NONLINEAR PROPAGATION}

In this section, we briefly investigate how the resonance observed in the linear model behaves when nonlinear effects are taken into account. The previous problem is now solved numerically with the nonlinear version of the Misthyc code.

First, the deformation is kept linear, i.e. with a very small ground deformation amplitude: $\zeta_{0}^{*}=0.001$. As done with the linear theory, the maximal amplitude ratio $\eta^{*} / \zeta_{0}^{*}$ is calculated at the end of the ground motion for the same range of parameters $\left(v_{p}^{*}\right.$ and $\left.\tau^{*}\right)$. The spectrum is shown in Figure 7 and is very similar to the one from the linear theory in Figure 3. Then, the nonlinearities are introduced by increasing the amplitude of the sea floor deformation $\zeta_{0}^{*}$ from 0.001 to 0.1 . The free surface deformation is only computed for $v_{p}^{*}=1$ and $\tau^{*}=0$ and is plotted in Figure 8 at the end of the ground motion.

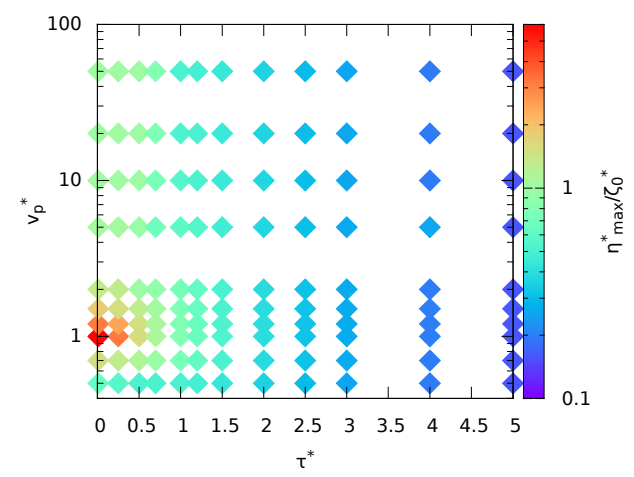

Figure 7: The maximum free surface amplitude $\left(\eta_{\max }^{*} / \zeta_{0}^{*}\right)$ calculated with the nonlinear numerical model as a function of $v_{p}^{*}$ and $\tau^{*}$ for $L^{*}=50$ at $t^{*}=t_{r}^{*}+L^{*} / v_{p}^{*}$ (colour in $\log$ scale).

The numerical results for $\zeta_{0}^{*}=0.001,0.005,0.01$ are close to the linear solution. The general wave form is preserved, and the maximum amplitude increases slightly with $\zeta_{0}^{*}$. For $\zeta_{0}^{*}=0.05$ and 0.1 , the propagation is faster. The frequency dispersion is more pronounced, and the first generated wave seems to have split already in two for $\zeta_{0}^{*}=0.1$, leading to a lower maximum amplitude than observed in the other cases. 


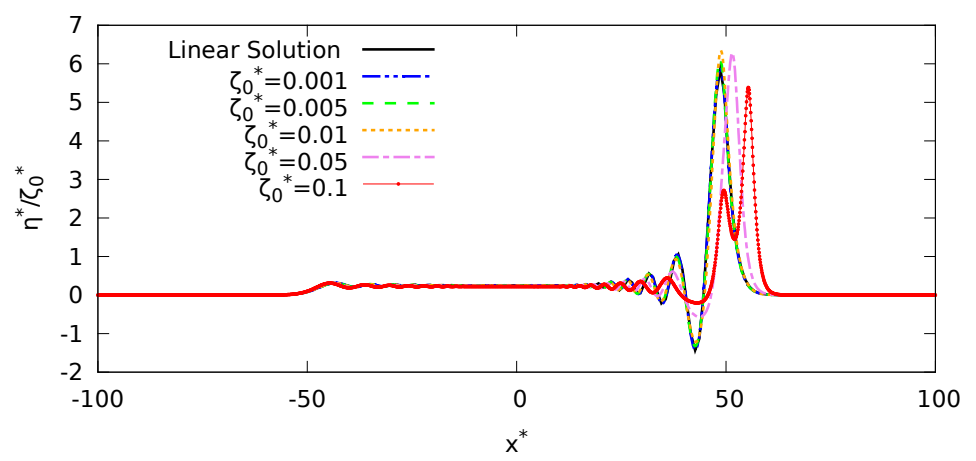

Figure 8: Deformation of the free surface at $t^{*}=T^{*}$ and for $v_{p}^{*}=1$ and $\tau^{*}=0$ : comparison of the linear solution (black line) and nonlinear numerical results. The coloured dashed lines represent the dimensionless nonlinear numerical results for different increasing nonlinearity levels: $\zeta_{0}^{*}=0.001,0.005,0.01,0.05,0.1$.

In reality, this kind of deformation $\left(\zeta_{0}^{*}=0.05\right.$ and 0.1$)$ is very large for seismic tsunami generation: the ground deformation is usually on the order of $10 \mathrm{~m}$ for a water depth of $4000 \mathrm{~m}$, leading to $\zeta_{0}^{*}$ on the order of $10^{-3}$.

Finally, for every value of $\zeta_{0}^{*}$, the wave generated is larger than the deformation's amplitude. Thus, the resonance is not restricted to a linear framework. However, from a certain level of nonlinearity, the dispersion phenomenon appears faster, that leads to smaller maximal wave amplitude.

\section{CONCLUSION}

A linear semi-analytical solution is built and validated for the free surface deforamtion generated by a simplified motion of the sea ground. This motion is function of $t_{r}$ (the rise time) and $v_{p}$ (rupture propagation velocity). We studied the influence of the timescales by estimating the amplification ratio, $\eta_{\max }^{*} / \zeta_{0}^{*}$, between the maximal amplitude of the free surface deformation and the final ground deformation amplitude. The spectrum of the amplification ratio shows that a resonance exists for rupture velocities close to the linear wave celerity $(\sqrt{g h}$, as emphasized by Todorovska and Trifunac (2001)) and short rise times. This resonance manifests as high ratio $\eta_{\max }^{*} / \zeta_{0}^{*}$ that can be greater than 1 . The value of the ratio is function of the deformation length, an empirical relation suggests a power law. Moreover, numerical simulations showed that this resonance is not restricted to the linear theory and is still present within a nonlinear framework even if frequency dispersion decreases the maximal free surface amplitude for the largest $\zeta_{0}^{*}$.

\section{ACKNOWLEDGEMENTS}

This work was funded by the French project "Tsunamis in the Atlantic and the English ChanNel: Definition of the Effects through numerical Modelling" (TANDEM). The authors would like to thank Marissa Yates and Cécile Raoult from the Saint-Venant Hydraulics Laboratory (France) for their help with the program Misthyc.

\section{References}

D. Dutykh, D. Mitsotakis, X. Gardeil, and F. Dias. On the use of the finite fault solution for tsunami generation problems. Theoretical and Computational Fluid Dynamics, 27:177-199, 2013.

J. Hammack. A note on tsunamis: their generation and propagation in an ocean of uniform depth. Journal of Fluid Mechanics, 60:769-799, 1973.

T. Jamin, L. Gordillo, G. Ruiz-Chavarría, M. Berhanu, and E. Falcon. Experiments on generation of surface waves by an underwater moving bottom. Proceedings of the Royal Society of London A: Mathematical, Physical and Engineering Sciences, 471(2178), 2015.

H. Kanamori. Mechanism of tsunami earthquakes. Phys. Earth Planet. Interiors, 6:346-359, 1972. 
M. Nosov. Tsunami waves of seismic origin: The modern state of knowledge. Izvestiya, Atmospheric and Oceanic Physics, 50(5):474-484, 2014. ISSN 0001-4338.

Y. Okada. Internal deformation due to shear and tensile faults in a half-space. Bulletin of the Seismological Society of America, 82, № 2:1018-1040, 1992.

M. I. Todorovska and M. D. Trifunac. Generation of tsunamis by a slowly spreading uplift of the sea floor. Soil Dynamics and Earthquake Engineering, 21:151-167, 2001.

M. L. Yates and M. Benoit. Accuracy and efficiency of two numerical methods of solving the potential flow problem for highly nonlinear and dispersive water waves. International Journal for Numerical Methods in Fluids, 77(10):616-640, 2015.

V. E. Zakharov. Stability of periodic waves of finite amplitude on the surface of a deep fluid. Journal of Applied Mechanics and Technical Physics, 9:190-194, Mar. 1968. 